\title{
TECHNIQUE ET NOUVELLE SENSIBILITÉ DANS L'CEUVE DE HERBERT MARCUSE
}

\author{
JULIANO BONAMIGO FERREIRA DE SOUZA
}

\begin{abstract}
The article focuses on the role played by the critique of technology in the thought of German philosopher Herbert Marcuse (1898-1979). We argue that Marcuse has developed not only an important criticism of modern technology but also an original theory on the new possibilities of technique, based on the concept of new sensibility, developed mainly in the books An Essay on Liberty (1969) and Counterrevolution and Revolt (1972). On the basis of this hypothesis, our essay first pays particular attention to Marcuse's analysis of instrumental rationality, where we show that according to the author's perspective, despite technology's use for instinctive domination, it carries emancipatory possibilities. In the second part, we analyse the Economic and Philosophic Manuscripts of 1844, from Karl Marx, pointing that his proposition of a freed sensibility in human's relation to nature deeply contributes to Marcuse's perspective of emancipation. Finally, we analyse Marcuse's thesis developed during the 1960's and 1970's, showing how the author builds the proposition of new sensibility in relation to emancipatory possibilities of imagination, arguing that his analysis leads to a new form of science and to a new approach to nature.
\end{abstract}

\section{Introduction}

Le travail philosophique de Herbert Marcuse (1898-1979) comprend une analyse importante du rôle de la science et de la technique au sein de la société industrielle. Après Éros et civilisation (1955), ouvrage dans lequel l'auteur établit les grandes lignes du freudo-marxisme, l'analyse des modes de subjectivation sous le système économique capitaliste se fait à partir d'une dimension pulsionnelle, et la réification est analysée non seulement par son enracinement dans le travail aliéné, 
mais aussi par rapport aux mécanismes répressifs opérants au sein des modes de production contemporains. En procédant d'une telle manière, en même temps que des auteurs comme Max Horkheimer et Theodor W. Adorno, Marcuse parvient à dévoiler l'instrumentalisation de la raison qui ordonne les modes de vie dans la société capitaliste, soulignant ainsi non seulement les principaux aspects répressifs de la chaîne productive, mais aussi la manutention d'un principe de réalité responsable du façonnement du cadre anthropologique pulsionnel. ${ }^{1}$

La critique de la société industrielle avancée et de l'instrumentalisation de la raison qui se manifeste dans son intérieur, telle que mise en œuvre par Marcuse, dévoile une technique soumise aux enjeux historiques et politiques. En ce sens, la dernière période de l'œuvre de Marcuse présente une discussion autour d'une société rationnelle qui ne pourrait se réaliser qu'à travers une dimension esthétique, tout en privilégiant les possibilités émancipatrices de la technique. ${ }^{2}$ L'imagination et le recours à l'aisthesis ${ }^{3}$ apparaissent alors comme la voie d'accès privilégiée à ces possibilités utopiques et émancipatrices, et pouvant régir l'usage technique et l'engendrement mutuel de l'homme et de la nature dans son rapport réciproque. ${ }^{4} \mathrm{Le}$ concept de nouvelle sensibilité, présenté dans Vers la libération (1969) est, en ce sens, le fil conducteur de notre analyse.

Afin de défendre l'hypothèse selon laquelle ce parcours esthétique est fondamental au sein de l'œuvre de Marcuse, nous procèderons en trois temps. Dans un

1 Le livre Éros et civilisation. Contribution à Freud, écrit en 1955, marque un ralliement de Marcuse à la pensée de Freud, qui conjointement à celle de Marx donne lieu au freudo-marxisme présent dans son œuvre. À partir de cette liaison, l'analyse politique marcusienne comprend la subjectivité comme étant structurée autour d'une nature instinctuelle, composée de pulsions enracinées dans le substrat biologique humain. Ainsi, une partie importante du diagnostic de Marcuse utilise la notion psychanalytique de principe de réalité. Cela signifie, dans le cadre de l'argument de l'auteur, la structure symbolique et matérielle qui façonne le statu quo social. Marcuse réactualise ce concept en montrant comment les processus productifs contemporains imposent aux individus une contrainte performative, intensifiant ainsi les processus de répression et d'aliénation à l'intérieur du système capitaliste.

2 Ce que nous délimitons ici comme étant la dernière période de l'œuvre de Marcuse comprend les ouvrages suivants : L'Homme unidimensionnel (1964), Vers la libération (1969), Contre-révolution et révolte (1972) et La dimension esthétique (1977).

3 La notion d'aisthesis, qui sous-tend la dimension esthétique de l'auteur, renvoie précisément aux modes de sensibilité et aux possibilités des sens. Une deuxième couche sémantique du terme esthétique, qui implique la production et la jouissance d'œuvres d'art, se trouve également dans les œuvres de Marcuse.

4 Reitz, Charles: Art, Alienation and the Humanities. A Critical Engagement with Herbert Marcuse, SUNY Press, New York 2000, pp. 101ff. Marcuse pense l'imagination en s'inspirant de la philosophie critique de Kant. Dans la tradition philosophique précritique, l'imagination a souvent été comprise comme reproductrice du réel - en le déformant souvent -, alors que chez Kant l'imagination peut contribuer à transformer le réel, s’affirmant comme pouvoir de déterminer la sensibilité. 
premier moment, nous exposerons les principaux points de l'analyse de la raison instrumentale et technique à partir des textes de Marcuse sur Max Weber. Dans un second moment, nous explorerons la thèse d'une transformation de la sensibilité inscrite dans les textes du jeune Marx et qui se trouve reprise par Marcuse, en montrant en quoi cette lecture est à la genèse de l'idée d'une dimension esthétique. Enfin, nous montrerons comment l'auteur parvient à construire la thèse d'une dimension esthétique en inscrivant en son centre le concept de nouvelle sensibilité, afin de mettre l'imagination au service d'un projet technologique d'émancipation.

\section{Critique de la rationalité instrumentale}

Depuis 1944, Max Horkheimer et Theodor W. Adorno soulignaient la complexification de la réification infligée aux individus par le processus technique dominant de la société industrielle avancée, dans laquelle la raison organisatrice des modes de vie était devenue une " auxiliaire de l'appareil économique » qui englobe toute la vie sociale..$^{5}$ Dans la même voie, Horkheimer, dans Éclipse de la raison (1947), écrivait que « la rationalisation de plus en plus avancée telle qu'on la comprend et qu'on la pratique dans notre civilisation tend [...] à détruire la substance même de la raison au nom de laquelle on adhère au progrès ${ }^{6}{ }^{6}$

Cette critique des déviances de la raison, partagée par la majorité des auteurs de la première génération de l'École de Francfort, constitue le fondement d'un projet critique dans l'ouvre de Herbert Marcuse. Ce projet, formulé depuis les années 1940, se développe comme réflexion à l'égard des aspects émancipateurs de l'esthétique. Face à la contradiction instaurée par la civilisation industrielle avancée - de plus en plus performante dans la production de l'abondance, mais assujettie par les contraintes d'une rationalité instrumentalisante, qui détermine l'ensemble de la vie par la planification et par une rationalisation de plus en plus croissante ${ }^{7}$-, l'œuvre de Marcuse se différencie ainsi par l'attention portée à la rencontre entre esthétique et technique, et à ses potentialités émancipatrices.

Afin de clarifier ces propositions, il est important de revenir aux premières analyses faites par Marcuse concernant le phénomène de la rationalisation technique,

5 Horkheimer, Max et Adorno, Theodor W. : La dialectique de la raison, trad. fr. Éliane Kaufholz, Gallimard, Paris 1974 [1947], p. 59.

6 Horkheimer : Éclipse de la raison, trad. fr. Jacques Laizé, Payot, Paris 1974 [1947], p. 10.

7 Marcuse, Herbert: «Some Social implications of Modern Technology ", in Studies in Philosophy and Social Science, Vol. 9, Nr. 3, 1941, pp. 414-439. Ces caractéristiques appartiennent au concept de rationalité technique tel qu'analysé par Marcuse. La réflexion de Marcuse à l'égard du surgissement de ce nouveau type de rationalité remonte à cet essai de 1941 . 
notamment dans un essai de 1964, consacré à l'œuvre de Max Weber. Selon lui, " la rationalité occidentale devient rationalité économique du capitalisme », ce qui entraîne la subsistance d'une "recherche incessante du profit par le fonctionnement continu et rationnel de l'entreprise capitaliste » dans les modes de production régnants au sein de la civilisation industrielle ${ }^{8}$. Fonctionnant à partir d'une dynamique d'abstraction, cette rationalité réduit toute qualité en quantité, par une réduction à une nécessité de fonction ou de valeur d'échange économique. En fin de compte, cette raison, définie comme raison technique, en vient à se concrétiser sous forme de domination "calculable et calculée, domination sur la nature et sur les hommes ". ${ }^{9}$ La dynamique propre à cette rationalité, que Marcuse va chercher dans l'œuvre de Weber, détermine alors l'angle d'approche à partir duquel sa théorie critique pourra discerner l'état de la technologie à l'intérieur de l'appareil productif contemporain.

L'aspect le plus frappant de cette opération est celui permettant à dévoiler les déterminations historique et politique au sein desquelles cette rationalité apparait. Cela indique la structure idéologique responsable par le caractère nocif de la technique. En effet, la lecture de Weber rappelait déjà que cette raison technique obéit à un ordre politique, et qu'elle se maintient dans les limites fixées par "l'intérêt de cette domination sur les hommes ". Son aspect politique est historique, et la servitude qu'elle instaure n'est donc aucunement une nécessité, puisque « la raison technique est toujours la raison du système social de domination ; elle peut être changée dans sa structure même $»{ }^{10} \mathrm{Et}$ dans la mesure où cela n'est pas une nécessité interne à la technique, on révèle sa possibilité libératrice, malgré le stade d'instrumentalisation dénoncé par Marcuse. ${ }^{11}$

C'est la nécessité de dévoiler l'aspect historique, voire idéologique d'une telle structure de domination qui est en jeu dans la réactualisation de Weber par Marcuse. Autrement dit, l'asservissement de la technique serait imposé par le milieu politique dans lequel cette technique s'exerce, ce qui par conséquent permet de la penser dans un façonnement historique de ses forces productives. Cette détermination historique permet à la critique marcusienne de cibler la rationalisation du capitalisme, sans pour autant avoir à fermer la voie à une assimilation positive et utopique des avancées techniques de la civilisation industrielle. Car si la technique

8 Marcuse : «Industrialisation et capitalisme chez Max Weber », in : Culture et société, trad. fr. Billy, Gérad; Bresson, Daniel et Graset, Jean-Baptiste, Les Éditions de Minuit, Paris 1965, p. 275.

9 Ibid.

$10 \quad$ Ibid., p. 293.

11 Marcuse: «The Responsibility of Science ", in: Krieger, Leonard et Stern, Fritz (Ed.), The Responsibility of Power: Historical Essays in Honor of Hajo Holborn, Double Day, Garden City (ny) 1967, pp. 439-444. 
est à chaque fois un projet historique et social, la raison technique qui l'ordonne "relève de l'idéologie », et la trame sociale dans laquelle les acquis scientifiques et techniques servent à la domination ne font que refléter « les intentions que nourrissent une société et les intérêts qui la dominent sur les hommes et les choses ». ${ }^{12}$ Cette approche est à la base de la compréhension marcusienne de la science et de la technique : celle-ci se trouve toujours prise à l'intérieur d'un cadre de domination sociale et d'un projet historique, dont l'orientation est déterminée par le règlement idéologique des forces politiques et productives dominantes au sein de l'être social occidental. Ce règlement, selon la spécificité de la critique matérialiste de Marcuse, apparaît comme la domination des classes fondée sur la propriété privée et sur les moyens de production, dont le pivot est la marchandisation du travail humain.

A y regarder de plus près, cette critique a toujours accompagné l'œuvre de Marcuse. On la voit par exemple à l'œuvre dans L'Homme unidimensionnel (1964) : la logique d'accumulation du capital et l'idéologie du progrès fondé sur ce système économique obligent à une manutention des contraintes pulsionnelles façonnées par la raison instrumentale. En dépit de ce diagnostic, et contrairement à ce que le contenu plus explicite de ses appréciations pourrait laisser entendre, la thèse de Marcuse indique bien plutôt une espérance portant sur les apports libérateurs de la technologie. Cela est dû au fait que la méthode scientifique à la base de l'assujettissement humain ne fait autre chose que d'encadrer les limites imposées par la Lebenswelt au sein de laquelle elle se trouve en marche. ${ }^{13}$ En ce sens, les résultats de la science ne sont que les produits du projet de domination sur la nature et sur l'humain lui-même - ce qui permet de revendiquer l'apparition d'une autre technique, dès que les conditions de possibilité de ce changement qualitatif sont présentes. Selon Marcuse,

s'il y avait un changement dans le sens du progrès qui briserait le lien entre la rationalité de la technique et celle de l'exploitation, il y aurait également un changement dans la structure même de la science - [...] et par conséquent la science aboutirait à des concepts de la nature essentiellement différents, elle établirait des faits essentiellement différents. ${ }^{14}$

\footnotetext{
Marcuse : «Industrialisation et capitalisme chez Max Weber », op. cit., p. 291.

13 Marcuse : L'homme unidimensionnel. Essai sur l'idéologie de la société industrielle avancée, trad. fr. Monique Wittig, Les Éditions de Minuit, Paris 1968 [1964], p. 188. Pour entreprendre une analyse de mathématisation du réel qui se trouve au cœur du rationalisme occidental, Marcuse mobilise notamment Edmund Husserl.

14 Ibid., pp. 189-190.
} 
La négativité de ce possible à l'égard de l'état actuel de la science montre que la critique de la planification de la vie et des effets de l'instrumentalisation de la raison - non seulement envers la nature extérieure, mais aussi envers la nature intérieure de l'humain -, ne se limite nullement à la simple dénonciation de la rationalité actuelle. On y voit bien plus les moyens possibles de libérer une rationalité autre. ${ }^{15}$ Mais si, dans cet enfermement causé dans et par la raison elle-même, l'organisation rationnelle des forces productives s'est montrée incapable d'achever les fins dernières de la vie, et si l'idée d'une utilisation plus rationnelle des ressources disponibles ou d'une réforme rationnelle dans les institutions sociales s'est avérée insuffisante, comment Marcuse dessine-t-il alors, à partir de l'explicitation de la structure oppressive, un horizon d'émancipation duquel une rationalité autre peut émerger ?16

Afin de mieux comprendre la proposition libératrice de Marcuse, il convient de garder à l'esprit le prisme psychanalytique à travers lequel l'auteur cherche à aborder les défis de l'instrumentalisation de la science et de la technologie, et qui est incorporé dans son œuvre à partir de la fin des années $1950 .{ }^{17}$ Tout au long de ce parcours, sa pensée se bâtit sur un fondement anthropologique pulsionnel, hérité de la rencontre entre l'œuvre de Marx et de Freud, marquant ainsi sa critique des modes de subjectivation propres à la réification dans le système économique capitaliste. La libération pulsionnelle proposée par Marcuse est réalisable par le biais esthétique et cherche à contrer la persistance des modes de rationalisation de la domination, qui obéissent à un principe performatif propre à la société industrielle avancé. ${ }^{18}$

Une voie esthétique, bien que déjà présente en germe dans les écrits antérieurs de Marcuse, atteint des contours plus clairs dans l'ouvrage Vers la libération (1969). Cela ne signifie pas que le chemin de l'émancipation consisterait en l'abandon d'une planification rationnelle de la vie, mais bien plutôt qu'il faut prendre comme point de départ la dimension esthétique, puisque c'est à travers elle qu'un

15 Nous verrons par la suite que, pour Marcuse, ces moyens consistent dans un changement dans l'approche technique et scientifique de l'homme envers la nature.

16 Raulet, Gérard : Herbert Marcuse. Philosophie de l'émancipation, Presses Universitaires de France, Paris 1992, p. 139.

17 Cette proposition est supportée par l'idée d'une dimension esthétique, qui apparaît pour la première fois en 1955, dans le livre Eros et civilisation. Nous en traiterons dans la troisième section de cet article, mais il est possible d'anticiper que le choix d'une voie esthétique face aux excès d'une raison instrumentalisante ne signifie pas un abandon complet des apports de la Raison pour la construction d'une société non-répressive. Bien plutôt s'agit-il alors de réaffirmer l'importance de la sensibilité et de l'imagination dans une construction sociale à la capacité d'être vraiment rationnelle.

18 Marcuse : Éros et civilisation, trad. fr. Jean-Guy Nény et Boris Fraenkel, Les Éditions de Minuit, Paris 1963 [1955], p. 50. 
changement sensible peut s'instaurer, ouvrant ainsi la voie à une transformation qualitative de la technique. Cette dimension apparaît donc comme un biais possible pour faire face aux impasses d'une science idéologiquement déterminée par les impératifs issus du système économique et politique. L’effort est donc ici celui d'une négation de l'immédiat - au sous-sol de la société industrielle, avec tout son pouvoir créatif, demeure un tissu social potentiellement libéré du travail nécessaire et aliénant. Mais selon Marcuse, cette utopie concrète ne verra pas le jour si l'on persiste dans une organisation strictement rationnelle des forces productives. ${ }^{19}$ Dans une société dans laquelle ces mêmes forces productives sont déjà capables de permettre la liberté de l'existence humaine sur terre, la persistance de cette raison est devenue rétrograde, précisément parce qu'elle n'est rien de plus que l'organisation et le maintien d'une structure de répression pulsionnelle. En d'autres termes, le caractère conservateur et contradictoire de cette société se traduit par le fait qu'elle a d'un côté développé son pouvoir de production à un niveau pouvant permettre la libération du travail nécessaire et aliéné, mais que, de l'autre côté, l'assujettissement au travail et l'accroissement des inégalités sociales et de la pénurie y demeurent toujours. Le fait qu'un éclatement de cette domination soit possible à travers la technologie et la chaîne productive disponibles dans le présent, rend évident que la perpétuation de cette contrainte est la perpétuation même d'un principe de réalité spécifique, appelé par Marcuse le "principe de rendement » [performance principle] - celui d'une société orientée vers le gain et la concurrence, dont l'expansion exige une « domination de plus en plus rationalisée ${ }^{20}$ - et qui, finalement, n'aurait plus de raison d'exister. Pour le dire plus exactement : il s'agit là d'une aporie face à laquelle la raison ne peut rien faire, puisqu'elle se trouve dorénavant instrumentalisée. La raison y est même impliquée, et l'originalité du diagnostic de Marcuse concernant l'unidimensionnalité réside dans le fait qu'il affronte la « vanité d'une solution dialectique de ce qui n'est plus une contradiction mais une neutralisation $» .^{21}$

19 Marcuse : Vers la libération. Au-delà de l'homme unidimensionnel, trad. fr. Jean-Baptiste Grasset, Les Éditions de Minuit, Paris 1969, p. 12 : « [1] es forces techniques et technologiques du capitalisme et du socialisme avancé recèlent des possibilités qui sont proprement utopiques [...]. Mais nous savons désormais que ni l'utilisation rationnelle de ces forces, ni [...] leur contrôle collectif par [...] (les ouvriers) ne suffiraient à supprimer la domination et l'exploitation ».

20 Marcuse : Éros et civilisation, op. cit., pp. 50 et $119 f f .$. Comme le rappelle Marcuse, « le progrès même de la civilisation sous le principe de rendement est parvenu à un tel niveau de productivité qu'on pourrait considérablement réduire la demande sociale d'énergie instinctuelle à consacrer au travail aliéné ; par conséquent, il semble que ce soit moins la 'lutte pour l'existence' qui exige le maintien de l'organisation répressive des instincts, que l'intérêt qu'il y a à prolonger cette lutte, que l'intérêt de la domination».

Raulet: Herbert Marcuse. Philosophie de l'émancipation, op. cit., p. 138. 
À la fin des années 1960, c'est face à cette promesse échouée de la rationalisation de l'organisation de la vie sociale que le projet philosophique marcusien essaie de trouver une réponse qui emprunte une voie esthétique, et qui s'appuie sur les forces libératrices de l'imagination. Avant d'entreprendre une analyse plus profonde du projet marcusien, pour lequel la spécificité de la nouvelle sensibilité est de permettre une nouvelle technique et une nouvelle relation avec la nature dans les modes de production, revenons aux Manuscrits économico-philosophiques de 1844 de Marx, dans la mesure où ils se révèlent fondamentaux pour la compréhension de l'hypothèse libératrice adoptée par Marcuse dans ses derniers écrits.

\section{Le fondement marxiste d'un nouveau rapport avec la nature}

Parallèlement à la critique de la rationalité technique et instrumentale, Marcuse accorde une attention particulière à la nécessité d'une transformation de la dimension sensible des individus, afin de permettre la mise en œuvre des transformations sociales effectives. Une des principales influences de ce principe sont les écrits du jeune Marx, qui ont été incorporés par Marcuse à partir des années 1930, et qui continuent à opérer même à l'intérieur de son intérêt pour la dimension esthétique.

Ce retour au jeune Marx est à la base du concept de nouvelle sensibilité, lorsque Marcuse reprend son analyse en 1972 dans son livre Contre-révolution et révolte, pour penser " une nouvelle relation entre l'homme et la nature ».22 Il est certain que cette proposition porte une critique à la manière par laquelle le progrès scientifique et technique met en œuvre une relation de consommation avec l'environnement. Pourtant, l'idée d'une nouvelle sensibilité implique en même temps une révision du rapport être humain-nature, tout comme une révision de l'engendrement du genre humain lui-même. Selon l'argument marcusien, une nouvelle sensibilité exige qu'une nouvelle relation avec la nature soit mise en place. Cela déclenche un changement dans la façon de réaliser les techniques de production et les décisions quant aux priorités à développer par le complexe réseau technologique et scientifique à disposition dans la société industrialisée contemporaine. Cette libération de la nature devient finalement une alliée pour la libération humaine de ses propres mécanismes d'oppression, puisque la violation objective de la nature aggrave la condition humaine elle-même. Pour comprendre cette relation entre l'être humain et la nature - et afin de comprendre surtout

22 Marcuse : Contre-révolution et révolte, trad. fr. Didier Coste, Seuil, Paris 1973 [1972], p. 81. 
comment elle implique l'engendrement d'une "nature humaine » elle-même -, il est nécessaire de revenir sur les Manuscrits économico-philosophiques de 1844 de Karl Marx. ${ }^{23}$ Selon Marcuse, on trouve déjà dans les Manuscrits l'idée d'une libération des contraintes imposées par les modes de productions capitalistes, libération qui ne serait possible qu'avec une « émancipation complète de tous les sens et de toutes les qualités humaines ». ${ }^{24}$ Cette analyse inscrite dans l'œuvre du jeune Marx permet à Marcuse de penser qu'une fois que la société se trouve libérée des rapports de production centrés sur le travail aliéné et sur la propriété privée, le caractère anthropologique fondamental de l'activité d'objectivation permettra l'apparition d'un "nouveau type humain » capable de mettre en œuvre de nouveaux rapports de production et, par conséquent, une nouvelle science basée sur une nouvelle sensibilité.

Pour le Marx des Manuscrits, la propriété privée est le pivot autour duquel s'organise le travail aliéné. C'est pourquoi une grande partie des Manuscrits est dédiée à la dénonciation de l'organisation du travail qui engendre et soutient la propriété privée elle-même au sein de l'économie politique classique. Le fait paradoxal signalé par Marx consiste en ce que la réalisation du travail apparaît comme déréalisation [Entwirklichung] du travailleur dans le mode de production capitaliste. ${ }^{25} \mathrm{Le}$ produit du travail est objectivation [Vergegenständlichung] du travail lui-même, il est le travail qui s'est fixé dans l'objet et qui s'est fait chose. Ce fait de l'économie politique est explicite : le travailleur « devient d'autant plus pauvre qu'il produit plus de richesse, que sa production s'accroît en puissance et en extension ${ }^{26}$. Ainsi, l'analyse de Marx débute avec la constatation que dans le mode de production capitaliste basé sur la propriété privée, « le travailleur devient une marchandise au prix d'autant plus vil qu'il engendre plus de marchandises $» .{ }^{27}$ La réalisation du travail implique alors une déréalisation du travailleur, dans laquelle l'objectivation est donc de fait un asservissement à l'objet produit. Ce rapport explique bien la place du travail au sein de la structure de l'économie industrielle qui s'impose dans la production capitaliste « comme aliénation [Entfremdung], comme perte de l'expression [Entäußerung] ».28

23 Les Manuscrits économico-philosophiques de 1844 furent rédigés par Karl Marx lors de son séjour à Paris. Il s'agit d'un de ses principaux écrits de jeunesse, où on trouve Marx fort intéressé par la notion de travail humain et son rapport à l'économie politique.

24 Marx, Karl : Manuscrits économico-philosophiques de 1844, trad. fr. Franck Fischbach, Vrin, Paris 2014, p. 150 ; cf. également Marcuse : Contre-révolution et révolte, op. cit., p. 87.

25 Marx: Manuscrits économico-philosophiques de 1844, op. cit., p. 118.

26 Ibid., p. 117.

27 Ibid.

28 Ibid., p. 118. 
Le dévoilement de cette dynamique nocive dans l'appropriation du travail humain au sein de la production capitaliste est fondamental pour la critique marcusienne de l'état contemporain de la science et de la technique. Il permet en effet de montrer que le travailleur se rapporte au produit de son travail comme à un objet étranger, comme à une puissance indépendante de lui-même, alors qu'il en est le producteur : pendant que la puissance du monde qu'il aide à objectiver augmente, l'intérieur du travailleur se vide. C'est ainsi que Marx explique le processus d'objectivation qui, une fois inscrit dans la structure capitaliste, apparait dans un sens négatif comme un processus d'aliénation. Le monde naturel, duquel pourtant le travailleur tire sa subsistance, lui devient de plus en plus étranger dans la mesure où ce monde est consommé, s'anéantissant afin que le travailleur puisse assouvir ses nécessités physiques. Le produit du travail est donc la perte active de l'expression humaine, dans la mesure où ce travail n'est pas librement voulu, mais s'impose en tant que contrainte dans la structure productive, apparaissant ainsi plus comme souffrance que comme réalisation.

Pourtant cette aliénation n'est pas propre à la nature de l'activité humaine, mais le résultat historique de l'imposition des modes de production capitalistes. C'est pour cela que la relation à la nature à travers l'activité du travail est, pour le Marx des Manuscrits, une activité propre à l'humain. Aux yeux de la tradition suivie par Marx, le travail est une activité vitale, et sert les besoins les plus fondamentaux des animaux, l'humain inclus, en tant que vie générique. ${ }^{29}$ C'est pour cela que, selon Marx,

la vie productive est la vie générique. Elle est la vie qui engendre la vie. C’est dans la forme de l'activité vitale que repose le caractère entier d'une espèce, son caractère générique, et l'activité consciente et libre est le caractère générique de l'homme. ${ }^{30}$

Selon Marx, le travail serait naturel à l'être humain en tant qu'auto-activité, en tant qu'activité générique à travers laquelle l'humain s'engendre aussi bien luimême, que la nature qui l'entoure. Si le travail propre à l'humain a ainsi d'un côté pour caractéristique l'objectivation même de la vie générique, faisant en sorte que celui-ci s'intuitionne lui-même dans un monde produit par lui, la structure de la

29 Cf. Trân-vàn-Toàn : " Note sur le concept de "Gattungswesen" dans la pensée de Karl Marx », in Revue Philosophique de Louvain, Vol. 69, Nr. 4, Louvain-la-Neuve 1971, p. 527. Sous l'influence de L'essence du christianisme (1841) de Feuerbach, Marx affirme dans les Manuscrits de 1844 que l'être humain est un être générique [Gattungswesen]. Comme le souligne Trân-vàn-Toàn, "l'homme, en tant que Gattungswesen, est le travailleur conscient et social et [...] ne réalise son vrai être ainsi défini que dans un processus historique ".

Marx : Manuscrits économico-philosophiques de 1844, op. cit., p. 122. 
production capitaliste lui vole cette nature, faisant en retour qu'il soit aliéné de l'essence humaine [menschlichen Wesen entfremdet]. ${ }^{31}$ Le travail aliéné, c'est-à-dire le travail qui a perdu son expression [entäußerte Arbeit], apparaît alors comme une désappropriation de l'activité l'humaine. ${ }^{32}$ Contrairement à cela, le travail aliéné arrache l'humain à sa vie générique, l'empêchant ainsi d'élaborer son monde objectif, de coproduire sa réalité avec la nature.

Mais cette analyse de Marx ne sert pas seulement à Marcuse dans la mesure où elle fournit une base philosophique à la critique du travail, et donc du travail aliéné au sein des sociétés industrielles avancées. Car cette analyse révèle également un autre facteur, essentiel à l'idée de technique que Marcuse cherche à fonder, à savoir l'idée d'un engendrement mutuel entre l'être humain et la nature. Ainsi, l'humanisation de la nature implique également une naturalisation de l'homme. La nature devient véritablement « la base [Grundlage] " de l'existence humaine sociale, en ce qu'elle en est l'élément réel. ${ }^{33}$ Dans la description de Marx, naturalisme et humanisme se rencontrent. L'individu est un être social dont l'expression vitale [Lebensäußerung] est en même temps une expression de la vie sociale. La réalisation organique de l'humain et de ses sens, lorsqu'il s'objective ou qu'il s'approprie sa réalité, ou même lorsqu'il pâtit de quoi que ce soit, fait partie de ce que Marx appelle l'activation de la réalité humaine [Betätigung der menschlichen Wirklichkeit]. ${ }^{34}$ Cette activation se traduit dans un processus d'engendrement humain dans lequel son organicité et ses sens se forment et se façonnent conjointement avec la société et la nature humanisée. Ainsi,

c'est seulement par la richesse, objectivement déployée, de l'être de l'homme que, pour une part, sont formés et, pour une autre part, sont engendrés la richesse de la sensibilité humaine [menschlichen Sinnlichkeit] subjective, une oreille musicale, un œil pour

31 Ibid., p. 124.

32 Fischbach, Franck: Sans objet: capitalisme, subjectivité, aliénation, Vrin, Paris 2012, p. 152. Comme le souligne Franck Fischbach, la perte occasionnée par l'aliénation du travail n'implique pas seulement le produit objectivé du travail, mais aussi l'activité du travail en tant qu'activité vitale : « [...] la perte de l'objet n'est d'ailleurs pas uniquement la perte de l'objet produit, au sens où il est approprié par un autre que celui qui l'a produit : la perte d'objectivité subie par le travailleur aliéné est bien plus radicale que la seule perte de l'objet produit par lui. Ce qu'il perd, nous dit Marx, ce sont les objets nécessaires à la vie et les objets de travail [die Arbeitsgegenstände] : par où il faut entendre, d'une part, les objets nécessaires à la satisfaction des besoins vitaux [...], objets auxquels le travailleur n'a pas accès autrement qu'en travaillant, et d'autre part les objets requis par le travail lui-même [...] que le travailleur ne possède pas et auxquels il n'a accès que par l'intermédiaire de celui qui lui fournit du travail. De sorte que ce dont le travailleur aliéné est privé, c'est finalement du travail lui-même en tant qu’objet nécessaire à sa propre vie ». Ibid.

Ibid., p. 149. 
la beauté de la forme, bref, des sens capables de jouissances humaines, des sens qui se confirment en tant que forces essentielles humaines [menschliche Wesenskräfte]. ${ }^{35}$

Cet avenir commun dévoilé par Marx se base alors sur ce qu'il appelle une « coappartenance [Wesenhaftigkeit] essentielle de l'homme et de la nature $"{ }^{36}$ La nature et l'être humain s'appartiennent mutuellement, et c'est de leur rapport d'engendrement partagé que surgit l'histoire. Sous la plume de Marx, l'essence de l'humain gagne une particularité tout à fait remarquable, et qui nous permet de présumer que l'avenir et la mobilité de l'engendrement mutuel entre l'être humain et la nature sont possibles à partir de l'activité comme trait principal du fondement anthropologique. En d'autres termes, si l'activité apparaît bien comme l'essence humaine, cette dernière n'est pas pour autant permanente, mais s'engendre ellemême à travers le rapport mutuel qui rassemble l'humain et la nature. L'homme, affirme Marx, naît par lui-même, s'engendre par lui-même, et sa production est finalement le procès par lequel il se produit lui-même, en « humanisant la nature et en se naturalisant lui-même $»{ }^{37}$

Or, cette mobilité de la structure anthropologique, déjà inscrite dans les travaux de Marx, permet de mieux cerner la perspective au sein de laquelle Marcuse pense un changement du rapport être humain-nature. Marx apparaît donc ici comme une des sources majeures pour la revendication d'un nouveau rapport avec l'environnement, qui est à la fois un changement de la structure naturelle humaine et des modes d'appropriation de la nature. ${ }^{38}$ Cette mobilité, qui chemine une voie positive dans l'horizon utopique défendu par Marcuse, représente le fondement pour penser une nouvelle sensibilité qui s'engendre mutuellement avec une nouvelle technique, c'est-à-dire un nouveau rapport avec les forces productives, et que Marcuse développera dans ces derniers ouvrages, comme nous le verrons par la suite.

\footnotetext{
Ibid., p. 151.

Ibid.

Ibid., pp. 218-220.

8 La centralité du travail en tant que capacité d'auto-engagement humain, ainsi que d'une affection mutuelle avec l'environnement, a été largement abordée par Marcuse lors de son premier contact avec les Manuscrits de 1844. De manière pionnière, les thèses de Marcuse ont été développées dans l'article Neue Quellen zur Grundlegung des Historischen Materialismus, in Die Gesellschaft, Nr. 9, 1932, pp. 136-174. Pour une analyse approfondie des premiers écrits de Marcuse et de l'impact de Marx sur son œuvre nous permettons de renvoyer à un travail antérieur, $c f$. Ferreira de Souza, Juliano B. : Ontologie et politique dans les premiers écrits de Herbert Marcuse (Dissertation de Master), Faculté de philosophie, arts et lettres, Université catholique de Louvain, 2017.
} 


\section{Imagination et nouvelle sensibilité}

La possibilité d'une nouvelle relation entre l'être humain et la nature, suggérée par la lecture du jeune Marx, guide le parcours suivi par Marcuse dans ses écrits des années 1960 et 1970. Il ne s'agit pas d'un anéantissement des acquis et des réalisations scientifiques et techniques des sociétés existantes, mais bien plutôt du diagnostic qu'un changement qualitatif dans le mode d'opération de cette accumulation technologique est nécessaire ${ }^{39}$. Si l'on s'en tient au diagnostic de Marcuse, il est certain que la capacité technique de la production dans les sociétés industrielles avancées n'a servi qu'à la cause de l'exploitation. Cela se traduit par la perspective psychanalytique de la subjectivité adoptée par Marcuse, et grâce à laquelle il devient possible de comprendre l'élaboration et le maintien d'un principe de réalité responsable d'un mécanisme de blocage des possibilités humaines au sein des rapports productifs contemporains. En d'autres termes, la libération envisagée par Marcuse passe par un épanouissement des fonctions vitales du genre humain, c'est-à-dire par un épanouissement de sa nature pulsionnelle, qui jusque-là a été réprimée par les relations de travail et par les modes de subjectivation au sein de la société capitaliste.

Pour Marcuse, ce système économique et productif engendre, à travers la rationalité instrumentale qui l'organise, un façonnement des pulsions humaines qui est subi comme une intégration des besoins et des satisfactions aux impératifs économiques et de production, obligeant ainsi la stabilisation et la manutention de ce système lui-même :

L'économie dite de consommation et la politique du capitalisme des monopoles ont forgé à l'homme une seconde nature qui le lie à la forme marchande sur un mode libidinal et agressif. Le besoin de posséder, de consommer, de manipuler, de renouveler constamment tous les gadgets, appareils, engins, machines de toutes sortes qui sont offerts, et même imposés, aux individus, le besoin de s'en servir au risque même de sa vie, est devenu, au sens que nous avons défini précédemment, un besoin «biologique ». 40

39 Cf. Löwy, Michael: « Marcuse and Benjamin: The Romantic Dimension », in Telos, Nr. 44, 1980, pp. 25-33. La critique de la technique dans l'œuvre de Marcuse, qui vise l'état positif et instrumental de la science, subit parfois une accusation injustifiée de technophobie, résultat d'un romantisme inconsistant. Pour une lecture détaillée de l'influence historique du romantisme dans l'œuvre de Marcuse, qui permet d'échapper à ce genre de piège exégétique.

Marcuse : Vers la libération, op. cit., p. 22. 
Cette imposition indique un enracinement de la contre-révolution dans la structure pulsionnelle des désirs humains - comme une espèce de formation de deuxième nature -, qui ne trouve satisfaction qu'à l'intérieur de l'univers matériel déterminé par l'ordre productif établi. En d'autres mots, il s'agit d'une "adaptation profonde, 'organique', de l'individu à une société atroce mais rentable $»{ }^{41}$ D'après ce constat, une rupture avec le continuum de répression ne serait possible qu'au moyen d'un changement radical dans la "dimension biologique " humaine ${ }^{42}$, ce qui entraînerait une rejection des valeurs et des besoins tels qu'ils sont établis par l'ordre productif et consumériste du capitalisme. Simultanément, briser un tel arrangement réclame de même un changement qualitatif des modes de production capable de renverser le fondement matériel de la domination. Avec une telle fin, l'inversion aurait pour condition subjective une redéfinition des besoins humains ${ }^{43}$, et un changement dans les modes et conditions du travail. ${ }^{4}$

Il devient possible ici d'entrevoir la centralité de la notion de dimension esthétique pour la thèse de Marcuse : elle apparaît comme le socle producteur de nouveaux projets de société. Du fait que l'organisation pulsionnelle et la sensibilité forment la base de l'expérience humaine, elles peuvent devenir en même temps des agents de libération, dans la mesure où elles s'orientent " conformément aux impératifs des pulsions de vie», et modifient ainsi leur « orientation et leurs objectifs actuels $"{ }^{45}$ C'est ainsi que dans son livre Vers la libération, Marcuse esquisse une libération de la technique qui accorde un rôle majeur à la sensibilité :

La protestation politique, par le caractère total qu'elle revêt, s'étend à une dimension qui jusqu'alors était restée, en tant que dimension esthétique, essentiellement apolitique. Et les éléments ainsi activés par la protestation politique sont précisément les plus fondamentaux et organiques de cette dimension esthétique : la sensibilité humaine, en révolte contre les impératifs de la raison répressive et invoquant le pouvoir sensible de l'imagination. Une telle action politique, qui s'attache à une moralité et à une sensibilité nouvelles, à la fois comme conditions et comme résultats du changement social, se situe à un moment où la rationalité répressive qui a mené aux réalisations de la société industrielle devient parfaitement rétrograde, et n'est plus rationnelle que dans sa capacité d'endiguer' la libération. ${ }^{46}$

\footnotetext{
Ibid., p. 30 .

Ibid., p. 22.

Marcuse : L'homme unidimensionnel, op. cit., pp. 269-270.

Marcuse : Vers la libération, op. cit., p. 35.

Ibid., p. 43.

Ibid., p. 61.
} 
À partir d'une dimension esthétique, la revendication de Marcuse est de libérer l'être humain des contraintes économico-politiques qui ont forgé ses instincts et ses besoins à travers l'histoire. Pour cela, il devient nécessaire d'accéder à une nouvelle expérience du monde et d'autrui, qui échappe à cette dynamique d'exploitation, et qui, selon l'argument marcusien, n'est possible qu'après l'apparition d'une nouvelle sensibilité. Selon une structure empruntée à l'esthétique de Kant, cette nouvelle sensibilité entraîne un changement dans les modes par lesquels l'imagination interagit avec l'entendement. Échappant aux déterminations conceptuelles, l'imagination revêtirait ainsi un rôle clé dans la schématisation de nouvelles formes et de nouvelles possibilités d'action et de liberté dans la conformation du monde naturel et social. ${ }^{47}$ Par conséquent, les fins de la technique dans son contact avec le monde naturel changeraient, ce qui impliquerait une transformation de l'impact de la technologie sur la nature. Ainsi, la dimension esthétique, reléguée jusqu’alors à une sphère séparée de la vie sociale, doit désormais s'étendre à l'ensemble du champ de l'action. ${ }^{48}$

Ce nouveau cadre esthétique du projet marcusien dans Vers la libération permet à l'imagination d'être capable de façonner une technologie de la libération au sein de laquelle non seulement la nature, mais aussi la forme des objets à produire seront présidées par les pulsions de vie..$^{49} \mathrm{Ce}$ changement d'orientation autorise une technologie et une science qui protègent et enrichissent la vie, et qui se réalisent « en jouant librement des potentialités de la forme et de la matière $»{ }^{50} \mathrm{Ce}$ nouveau rapport entre sensibilité et imagination entraîne ainsi un nouvel ethos scientifique, selon lequel les sens sont producteurs et revêtent un rôle capital dans l'élaboration des images de la liberté, parce qu'ils fournissent à l'imagination, sous la forme d'expériences, le matériau à partir duquel celle-ci pourra construire le domaine de la liberté, devenant à la fois productrice et pratique. ${ }^{51}$

Ainsi, Marcuse propose une réalité façonnée par la dimension esthétique que ce soit par rapport aux sens ou par rapport à l'art -, dans laquelle « la sensibilité humaine, en révolte contre les impératifs de la raison répressive ", invoque le

47 Cette interaction est appelée jeu par Kant dans la Critique de la faculté de juger (1790), et a été fondamentale pour la constitution de la première proposition de dimension esthétique dans Eros et civilisation. Marcuse y entreprend une lecture de Kant et de Schiller et cherche à montrer comment la troisième Critique ouvre une voie de recherche philosophique permettant de penser les interconnexions entre esthétique, liberté et beauté au-delà des déterminations transcendantales de Kant. Cette interaction possible des facultés offre à Marcuse la possibilité de proposer une place privilégiée à l'imagination dans la construction d'une transformation sociale.

Ibid., p. 50.

Ibid., p. 38.

Ibid., p. 44 
pouvoir créateur de l'imagination, à la fois comme condition et comme résultat du changement social ${ }^{52}$ :

Au-delà des limites, au-delà du pouvoir de la raison répressive apparaît la possibilité d'un rapport nouveau entre sensibilité et raison, d'une harmonie entre la sensibilité et une conscience radicale, celle-ci consistant en des facultés rationnelles rendues capables de concevoir et de définir les conditions (matérielles) objectives de la liberté, ses limites réelles et ses chances d'aboutir. Mais alors, au lieu d'être conditionnée et imprégnée par la rationalité de la domination, la sensibilité serait guidée par l'imagination, qui effectuerait la médiation entre les facultés rationnelles et les besoins sensibles. ${ }^{53}$

Ce nouveau rapport permet à la transformation de la technologie d'ouvrir une voie pour l'abolition du travail aliéné, tout en restant une solution contre la pénurie matérielle. Celle-ci, au lieu de dépendre d'un rapport d'assujettissement de la nature, entrera en une relation d'engendrement mutuel avec elle. ${ }^{54}$ Dès lors le projet philosophique de Marcuse, qui cherche à établir une nouvelle relation pacifiée de l'homme et de la nature, prend tout son sens. ${ }^{55}$ L'affirmation selon laquelle seule une " libération complète de tous les sens et qualités humaines ${ }^{56}$ peut mener à la liberté - revendiquée par Marx dans les Manuscrits de 1844 et devenue une des sources théoriques de Marcuse, tel que nous l'avons montré dans la section antérieure - indique que c'est seulement suite à une libération et à un épanouissement des possibilités anthropologiques que l'instauration d'une nouvelle sensibilité - et par conséquent d'une nouvelle science - seront possibles. ${ }^{57} \mathrm{Il}$ s'agit d' " une nouvelle relation entre l'homme et la nature ${ }^{58}$ suite à laquelle l'imagination, unifiant entendement [Verstand] et sensibilité [Sinnlichkeit] et devenant ainsi pratique, sera capable de produire une science et une technologie pacifiées avec l'environnement. ${ }^{59}$ Marcuse réopère donc une structuration kantienne de l'imagination,

\footnotetext{
Ibid., p. 61.

Ibid., p. 46.

54 Ibid., p. 30. La technique et la science ne sont pas, pour Marcuse, des agents de domination en soi. C'est au contraire justement à travers elles qu'une libération du travail aliéné peut se faire, parce qu'elles peuvent représenter la fin de l'état de pénurie humain, dès lors qu'elles sont employées en dehors d'une utilisation restrictive de la société répressive. C’est dire que, pour Marcuse, «la science et la technologie sont les principaux agents de la libération ».

55 Marcuse : L'homme unidimensionnel, op. cit., pp. 258-259.

56 Marx : Manuscrits économico-philosophiques de 1844, op. cit., p. 150 ; Marcuse : Contre-révolution et révolte, op. cit., p. 87.

57 Kellner, Douglas: Herbert Marcuse and the Crisis of Marxism, Macmillan, London 1984, p. 338 sq.

58 Marcuse : Contre-révolution et révolte, op. cit., p. 81.

59 Marcuse: Vers la libération, op. cit., p. 59.
} 
mais en la mettant au service d'un projet pratique qui nie la totalité telle qu'elle se présente, en cherchant à la transformer en allant à la rencontre de la liberté dans la nature. Par conséquent,

au lieu d'être conditionnée et imprégnée par la rationalité de la domination, la sensibilité serait guidée par l'imagination, qui effectuerait la médiation entre les facultés rationnelles et les besoins sensibles. La conception grandiose qui anime la philosophie critique de Kant fait voler en éclats le contexte philosophique dans lequel il l'a confinée. L'imagination, en unifiant la sensibilité et la raison, devient " productive » en même temps qu'elle devient pratique : elle devient une force motrice dans la reconstruction de la Lebenswelt - reconstruction assurée par une Gaya scienza, par une science et une technologie qui, ne servant plus la cause de l'exploitation et de la destruction, se trouveraient disponibles pour les exigences libératrices. La transformation rationnelle du monde pourrait alors aboutir à une réalité façonnée par la seule sensibilité esthétique de l'homme. Dans un tel univers, les facultés et les désirs de l'homme pourraient, littéralement, s'incarner, s'incorporer, au point d'apparaitre comme inclus dans le déterminisme objectif de la nature : congruence de la causalité naturelle et de la causalité libre. ${ }^{60}$

\section{Conclusion}

C'est ainsi au moyen de la dimension esthétique, des pulsions et de la sensibilité, que la thèse de Marcuse vise à une transformation effectivement rationnelle du monde - en y agençant simultanément une liberté dont la finalité technique respecte, avant tout, les besoins de la vie. On voit ainsi qu'une nouvelle sensibilité permet un nouvel ethos scientifique, et ce à l'intérieur même du projet de Marcuse. C'est bien à l'aide de l'imagination que Marcuse compte pouvoir faire émerger des modes de vie et des pratiques non encore réalisées, mais qui, en se réalisant, seront conformes aux besoins vitaux de l'humanité et qui achèveront ainsi, selon cette perspective, une " pacification de l'existence ». ${ }^{61}$ Comme nous avons pu montrer, la notion d'un engendrement mutuel entre l'être humain et la nature, idée déjà présente dans l'analyse des Manuscrits de 1844 de Marx et reprise par Marcuse dans la perspective adoptée au long des années 1960 et 1970, constitue une caractéristique centrale de cette thèse. À l'aide d'une matrice conceptuelle psychanalytique, cette idée se trouve alors réactualisée dans le désir de prendre

60 Ibid., p. 63.

$61 C f$. Feenberg, Andrew : « Marcuse et l'esthétisation de la technologie », in Quaderni, N. 49, Éditions de la MSH, Paris 2003, pp. 81-101. 
en compte les besoins vitaux au sein d'une coproduction technologique avec les formes de la nature. Simultanément, on entrevoit alors une matrice kantienne opérer à l'intérieur de ce projet : c'est bien de là que proviennent les idées d'une dimension esthétique et du rôle central accordé à la sensibilité dans l'élaboration d'un changement social. Les sens, ainsi libérés de l'a priori historique ${ }^{62}$ ayant causé l'assujettissement de la nature,

ne se contentent pas de 'recevoir' ce qui se présente dans les modes de vie contraignants, ils ne 'délèguent' pas la transformation du donné à une autre faculté - l'entendement. Au contraire, ils découvrent ou peuvent découvrir par eux-mêmes, dans leur 'pratique', de nouvelles, de plus réjouissantes possibilités et facultés, formes et qualités des choses, ils peuvent revendiquer et guider leur réalisation. ${ }^{63}$

Une nouvelle relation avec la nature permet ainsi de comprendre cette dernière comme un sujet doté de potentialités propres, au lieu de s'adresser simplement à l'environnement dans le but d'en tirer profit ou d'en extraire une matière première anodine et sans vie. ${ }^{64} \mathrm{Chez}$ Marcuse, on a affaire à un projet d'émancipation dont la condition sine qua non est de refuser toute assujettissement de la nature. Si la cause finale de la structure productive avait jusqu’à présent été déterminée par la seule quantification économique, tout en dissimulant le résidu nocif de sa rationalisation illimitée, la proposition inaugurée par Marcuse rejette cet approfondissement de la rationalisation, en raison des résultats atroces auxquels il aboutit.

Pour éclairer la structure formelle de ces thèses de Marcuse, nous avons essayé, tout au long des sections précédentes, de développer une hypothèse interprétative. Il était d'abord nécessaire d'expliciter et l'analyse proposée par la théorie critique, et le diagnostic auquel elle donne corps. Ce diagnostic a révélé une rationalité technique qui subsistait comme fondement de la structure productive analysée. C'est pourquoi il a fallu montrer comment Marcuse dissèque l'impact de cette structure productive sur la dimension subjective des individus. Celle-ci, en tant que socle anthropologique pulsionnel, souffre d'un façonnement du désir, sans pour autant perdre le fondement pulsionnel d'une vie toujours capable de se libérer. Dans un second temps, nous avons effectué un retour aux

62 Pour Marcuse, l'usage du concept d'a priori historique se réfère au déroulement économique qui débouche dans les modes de production capitaliste, conformées au long de l'histoire.

63 Marcuse : Contre-révolution et révolte, op. cit., p. 96.

64 Habermas, Jürgen : La technique et la science comme "idéologie ", trad. fr. Jean-René Ladmiral Gallimard, Paris 1973 [1968], pp. 11-12. Habermas affirme que, pour Marcuse, « le point de vue de la disposition technique qu'on peut avoir des choses ferait place à une attitude de sollicitude et de soin libérant les potentialités de la nature ». 
analyses marcusiennes des premiers écrits de Marx. Ces thèses, qui accompagnaient Marcuse depuis 1932, sont centrales pour comprendre l'importance de la sensibilité dans un projet de transformation sociale. Enfin, nous avons cherché à expliquer comment cette influence se complexifie et se cristallise dans l'idée d'une dimension esthétique, que Marcuse construit à partir de l'œuvre de Kant, et plus particulièrement grâce aux propositions esthétiques de la troisième Critique. Marcuse exploite ces catégories dans une formulation qui attribue à la sensibilité et à l'imagination des rôles centraux dans la construction de nouveaux modes d'interaction avec la nature - en incorporant son agentivité, afin de dessiner un projet technologique d'émancipation. ${ }^{65}$

Juliano Bonamigo Ferreira de Souza a étudié la philosophie à l'Université de São Paulo (2009-2014). En tant que boursier du programme EuroPhilosophie, il a ensuite étudié à l'Université de Toulouse et à l'Université catholique de Louvain, où il a obtenu son diplôme de master en 2017. Actuellement doctorant en philosophie au Centre de Philosophie du Droit (CPDR) de l'Université catholique de Louvain et boursier FRESH-FNRS, il développe un projet sur le rapport entre nature et technique dans l'œuvre de Herbert Marcuse.

65 Marcuse : Vers la libération, op. cit., p. 41ff.. Ce qui pourrait également être interprété comme une force productive sociale [gesellschaftliche Produktivkraft]. 